\title{
Modelos matemáticos baseados no Time Dependent Vehicle Routing Problem para planejamento da logística urbana sob a ótica ambiental
}

\author{
Bárbara Valente Ferreira ${ }^{1}$ e Rodrigo de Alvarenga Rosa ${ }^{2}$
}

\begin{abstract}
Resumo: Empresas que operam com logística urbana direcionam seus esforços para soluções que buscam reduções de custo, desconsiderando questões ambientais. Isso ocorre em função da crença que soluções ambientalmente corretas são mais caras. No entanto, com as crescentes preocupações ambientais, as empresas têm levado em conta os fatores ambientais buscando a responsabilidade social. Assim, este artigo apresenta dois modelos matemáticos, ambos baseados no Time Dependent Vehicle Routing Problem (TDVRP), sendo um com objetivo de avaliar a redução do tempo das rotas e o outro com objetivo avaliar a redução da emissão de poluentes. Para testar o modelo, foi realizada uma aplicação real de uma empresa de distribuição de alimentos na região metropolitana de Vitória, ES. Usou-se o CPLEX 12.6 para rodar os modelos propostos com cenários baseados em dados reais da empresa. Os resultados mostraram que a solução com viés ambiental pode ser financeiramente vantajosa para a empresa.

Palavras-chave: logística urbana, Time Dependent Vehicle Routing Problem, logística sustentável.
\end{abstract}

\begin{abstract}
Urban logistics companies are seeking solutions to reduce their cost, but must of them are not paying attention to environmental issues. This is due to the belief that environmentally friendly solutions are more expensive. However, with the growing of environmental concerns, companies have been taking into account the environmental factors, seeking for their social responsibility. Thus, this paper presents two mathematical models, both based on the Time Dependent Vehicle Routing Problem (TDVRP), one to evaluate the reduction in the time of the routes and the other to evaluate the reduction of greenhouse gas emissions. In order to evaluate the model, a real case of a food distribution company in the metropolitan area of Vitória, ES was done. CPLEX 12.6 was used to run both models considering scenarios based on data from a real company. The results showed that environmentally friendly solution may be also financially advantageous for the company.
\end{abstract}

Keywords: urban logistics, Time Dependent Vehicle Routing Problem (TDVRP), sustainable logistics.

\section{INTRODUÇÃO}

A logística urbana concentra-se, sobretudo, no planejamento da distribuição ou coleta de mercadorias em áreas caracterizadas por um forte adensamento de residências e atividades comerciais. $\mathrm{O}$ transporte efetuado nestas áreas, por conta deste adensamento e devido ao aumento do tráfego de veículos e pessoas, torna-se bastante complexo e com alto custo, além de gerar muitos impactos ambientais, dos quais se destaca a emissão de poluentes.

Empresas que operam com logística urbana costumavam focar seus esforços em soluções que trazem como retorno a economia financeira ou de tempo, desconsiderando as questões ambientais. Isso ocorria pela suposição de que aplicar soluções ambientalmente mais corretas ocasiona aumento dos custos para a organização. No entanto, nos últimos anos, com as crescentes preocupações ambientais, organizações industriais levam em conta fatores ambientais, a fim de melhorar o aspecto competitivo e buscam, cada vez mais, investigar estratégias e adotar aplicações da logística verde para considerar o uso preciso dos recursos naturais como uma responsabilidade social.

Desta forma, propõe-se neste artigo comparar os resultados de duas estratégias no transporte de mercadorias de

\footnotetext{
${ }^{1}$ Bárbara Valente Ferreira, Departamento de Engenharia de Produção,

UFES. (barbaravalentef@hotmail.com)

2 Rodrigo de Alvarenga Rosa, Mestrado em Engenharia Civil -

Transportes, UFES. (rodrigo.a.rosa@ufes.br)
}

Manuscrito recebido em 24/03/2016 e aprovado para publicação em 16/09/2016

Este artigo é parte de TRANSPORTES v. 24, n. 3, 2016. ISSN: 2237-1346 (online). DOI:10.4237/transportes.v24i3.1101 uma empresa de distribuição de alimentos de uma região metropolitana: uma cuja solução visa redução do tempo e outra cujo objetivo é diminuir a emissão de gases poluentes. Essa comparação possibilita avaliar a viabilidade de se considerar as questões ambientais nas soluções de problemas de transporte.

O fator mais preocupante no transporte de mercadorias nos problemas de logística urbana e que influencia profundamente em suas soluções é o congestionamento de veículos, que geralmente acontece nos horários de pico em que o número de veículos ultrapassa a capacidade do sistema viário, ocasionando desperdício de tempo, maior consumo de combustível e maior emissão de poluentes.

A fim de considerar o engarrafamento no planejamento da distribuição física, dentre os diversos conceitos de roteamento de veículos já estudados, o que mais se adere a esta realidade é o Time Dependent Vehicle Routing Problem (TDVRP). No TDVRP tem-se uma frota de veículos com capacidade limitada que deve coletar ou entregar cargas a clientes a partir de um depósito central. Os clientes devem ser designados aos veículos que realizam rotas, de forma que o tempo total gasto seja minimizado. O tempo de viagem entre dois clientes ou entre um cliente e o depósito depende de suas distâncias e também do momento do dia que o transporte é feito; por exemplo, nos horários de pico o tempo para deslocamento é maior devido ao congestionamento. As janelas de tempo para servir os clientes, ou seja, o período que os clientes podem ser atendidos, devem ser consideradas assim como a máxima duração permitida para cada rota (horário de trabalho do motorista) (Malandraki e Daskin, 1992). O TDVRP é, então, uma extensão do Problema de Roteamento de Veículos (VRP) que pode levar em 
conta o congestionamento urbano através de tempos de deslocamento que podem variar ao longo do tempo.

Mais especificamente, neste artigo foram propostos dois modelos matemáticos baseados no TDVRP denominados TDVRP-RT e TDVRP-RE. O TDVRP-RT é um modelo cuja função objetivo é reduzir a soma dos tempos até o regresso dos caminhões ao depósito. O TDVRP-RE foi proposto para solucionar Problema de Roteamento de Veículos Verde ou Green Vehicle Routing Problem (Lin et al., 2014, Erdogan et al., 2012) que tem por objetivo incorporar na sua função objetivo a redução das emissões de poluentes e adicionar às suas restrições questões ambientais.

Esse artigo está organizado em seis seções, a começar por essa introdução. Na Seção 2 tem-se uma breve revisão bibliográfica do TDVRP. A Seção 3 apresenta os modelos matemáticos propostos. Na Seção 4 é apresentada a metodologia, bem como as considerações na utilização dos dados da empresa. Na Seção 5 são apresentados e analisados os resultados obtidos. A Seção 6 é a seção final e apresenta as principais conclusões do artigo.

\section{REVISÃO BIBLIOGRÁFICA}

Para este artigo foi realizada uma revisão bibliográfica sobre o desenvolvimento dos problemas de roteamentos de veículos com dependência de tempo. Esta revisão é apresentada a seguir.

Hill e Benton (1992) e Malandraki e Daskin (1992) introduziram o conceito do TDVRP acrescentando ao VRP a variação de velocidade durante o dia devido às condições de trânsito. A função objetivo do TDVRP era minimizar o tempo de viagem, no entanto, dependia do momento do dia que o percurso era realizado e da ocorrência ou não de eventos aleatórios, como acidentes de trânsito. Fleischmann et al. (2004), Ichoua et al. (2003), Nannicini et al. (2010) e Van Woensel et al. (2008) aplicaram o conceito de First-In First-Out (FIFO) em seus modelos, evitando que um veículo fique parado em um nó esperando um melhor momento para viajar como foi sugerido por Malandraki e Daskin (1982).

Nos estudos desenvolvidos por Van Woensel e Vandaele (2006) e Van Woensel et al. (2008) buscou-se modelar o congestionamento por meio de dados empíricos de tráfego e do desenvolvimento de modelos de filas, resultando em velocidades médias para diferentes intervalos durante o dia. Palmer (2007) e Ericsson et al. (2006) estudaram as emissões no transporte de entregas a domicílio buscando relacionar o consumo de combustível e as emissões nos diferentes momentos do dia considerando a diminuição da emissão de $\mathrm{CO}_{2}$ como critério de otimização. A quantidade de gases de efeito estufa, de combustível, do tempo de viagem e seus custos foram calculados por Bektas e Laporte (2011), que incorporaram questões sobre poluição no VRP. Scott et al. (2010) investigaram a influência da redução da emissão de $\mathrm{CO}_{2}$ em instâncias pequenas.

As questões referentes ao fluxo de tráfego e seu impacto na emissão de poluentes foram analisadas por Van Woensel et al. (2001), Palmer (2007) e Kirby et al. (2000). Dentre os resultados alcançados pelos autores citados, destacam-se: o cálculo das emissões, quando o transporte é realizado considerando as velocidades constantes, leva a valores muito diferentes dos reais; a quantidade emitida de
$\mathrm{CO}_{2}$ pode aumentar em $40 \%$ durante períodos de congestionamento; e as emissões de $\mathrm{CO}_{2}$ são proporcionais ao consumo de combustível, sendo dependentes da velocidade e, portanto, do tempo.

A quantificação das emissões dos veículos depende de diversos fatores como idade e peso do veículo, estado e tamanho do motor, velocidade, tipo de combustível, etc. Assim, este cálculo tornasse muito complexo (Taniguchi et al. 2001). Jabali et al. (2012), além de adaptarem as funções de tempo propostas por Ichoua et al. (2003), considerando o conceito FIFO e as emissões em função da velocidade do modelo MEET, também sugeriram que fossem inseridos ao modelo outros tipos de emissões de poluentes.

\section{MODELOS MATEMÁTICOS PROPOSTOS}

O TDVRP é aplicado em casos de roteamento nos quais o tempo de viagem varia em função do momento do dia, então, o dia é dividido em intervalos de tempo. O problema é formulado em uma rede de nós onde cada nó representa um cliente e cada ligação representa o tempo de viagem de um nó a outro, e este tempo varia dependendo do momento do dia. A quantidade de ligações entre cada par de nós é determinada pela quantidade de intervalos de tempo que o dia foi dividido. Podem haver, por exemplo, três ligações devido a três intervalos do dia nos quais podem ser identificadas diferentes condições de trânsito - sendo o dia considerado um período de $8 \mathrm{~h}$ às $17 \mathrm{~h}$, o primeiro intervalo poderia durar $3 \mathrm{~h}$, o segundo $5 \mathrm{~h}$ e o terceiro $1 \mathrm{~h}$. No problema por Malandraki e Daskin, 1992, as operações podem ser de coleta ou distribuição e as cargas são coletadas ou distrubuidas por uma quantidade fixa de veículos homogêneos. O TDVRP não considera a possibilidade de ocorrerem ligações entre nós em diferentes intervalos de tempo. Um outro problema inerente ao TDVRP é que uma viagem pode começar em um período, mesmo que faltando um minuto para terminar o período, e o TDVRP considera que o restante da viagem ocorre neste período, mesmo tendo mudando de período. A função objetivo corresponde a minimizar o tempo total da rota de cada veículo, considerando restrições como a de capacidade máxima de carregamento de cada veículo, o momento mais cedo e o momento mais tarde para chegada do veículo ao cliente, (janela de tempo) e que em cada nó passará apenas um veículo e somente uma vez (Malandraki e Daskin, 1992).

Assim, o TDVRP-RT é o mesmo modelo proposto por Malandraki e Daskin (1992) que é composto pelos parâmetros:

$n$, o número de nós que representam os clientes/pontos a serem atendidos e também incluem o depósito de origem, 1 , e os depósitos virtuais, $n+1, \ldots, n+n v$, que representam os depósitos virtuais de retorno para cada veículo;

$m$, número de intervalos de tempo considerados para cada ligação entre dois nós distintos;

$n v$, número de veículos;

$t v_{i j}^{k}$, tempo de viagem do nó $i$ ao nó $j$ durante o intervalo

$k$, sendo ;

$t s_{i}$, tempo de serviço no nó $i$, sendo $t s_{i}=0$, parai $=1, n+1, \ldots, n+n v ;$ 
$T_{i j}^{k}$, limite superior para o intervalo de tempo $k$ para a ligação $(i, j)$;

ti, o momento mais cedo de partida dos veículos do depósito de origem, nó 1 ;

$\mathrm{cm}_{v}$, capacidade de carga (ou volume) do veículo $v$;

$c_{i}$, carga (ou volume) a ser entregue no cliente $i$, sendo $c_{i}=0$ para $i=1, n+1, \ldots, n+n v$;

$B$, número muito grande para a lógica do modelo;

$b$, capacidade do maior veículo;

$l_{i}$, momento mais cedo que o veículo pode chegar ao nó $i$;

$u_{i}$, momento mais tarde que o veículo pode chegar ao nó $i$.

O modelo compreende as seguintes variáveis de decisão:

$x_{i j}^{k}$, que assume valor 1 se algum veículo viaja diretamente

do nó $i$ para o nó $j$ partindo de $i$ durante o intervalo de tempo $k$ e o valor 0 caso contrário;

$t p_{i}$, momento de partida de algum veículo do nó $i$;

$q_{i}$, a quantidade acumulada de carga descarregada de um veículo quando parte do nó $i$.

A formulação matemática do TDVRP-RT pode ser escrita como:

$$
\operatorname{Min}\left(\sum_{v=1}^{n v} t p_{n+v}\right)
$$

sujeito às seguintes restrições:

$$
\begin{aligned}
& \sum_{\substack{i=1 \\
i \neq j}}^{n} \sum_{k=1}^{m} x_{i j}^{k}=1 \quad \forall j \mid j \in\{2, \ldots, n+n v\} \\
& \sum_{\substack{j=2 \\
j \neq i}}^{n+n v} \sum_{\substack{k=1 \\
m}}^{m} x_{i j}^{k}=1 \quad \forall i \mid i \in\{1, \ldots, n\} \\
& \sum_{j=2}^{n} \sum_{k=1}^{m} x_{1 j}^{k}=n v \\
& t p_{1} \geq t i \\
& t p_{j}-t p_{i}-B x_{i j}^{k} \geq t v_{i j}^{k}+t s_{j}-B \\
& \forall i, j, k \mid\left\{\begin{array}{l}
i \in\{1, \ldots, n\} \\
j \in\{2, \ldots, n+n v\} \\
i \neq j \\
k \in\{1, \ldots, m\}
\end{array}\right. \\
& t p_{i}+B x_{i j}^{k} \leq T_{i j}^{k}+B \quad \forall i, j, k \mid\left\{\begin{array}{l}
i \in\{1, \ldots, n\} ; \\
j \in\{2, \ldots, n+n v\} ; \\
i \neq j ; \\
k \in\{1, \ldots, m\}
\end{array}\right. \\
& t p_{i}-T_{i j}^{k-1} x_{i j}^{k} \geq 0 \quad \forall i, j, k \mid\left\{\begin{array}{l}
i \in\{1, \ldots, n\} ; \\
j \in\{2, \ldots, n+n v\} ; \\
i \neq j ; \\
k \in\{1, \ldots, m\}
\end{array}\right. \\
& l_{i}+t s_{i} \leq t p_{i} \leq u_{i}+t s_{i} \quad \forall i \mid i \in\{2, \ldots, n+n v\}
\end{aligned}
$$

$$
\begin{aligned}
& q_{j}-q_{i}-b \sum_{k=1}^{m} x_{i j}^{k} \geq c_{j}-b \quad \forall i, j \mid\left\{\begin{array}{l}
i \in\{1, \ldots, n\} ; \\
j \in\{2, \ldots, n+n v\} ; \\
i \neq j
\end{array}\right. \\
& q_{1}=0 \\
& q_{n+v} \leq c m_{v} \quad \forall v \mid v \in\{1, \ldots, n v\} \\
& t p_{i} \geq t i \quad \forall i \mid i \in\{1, \ldots, n\} \\
& q_{i} \geq 0 \quad \forall i \mid i \in\{1, \ldots, n\} \\
& x_{i j}^{k} \in\{0,1\} \quad \forall i, j, k\left\{\left\{\begin{array}{l}
i \in\{1, \ldots, n+n v\} ; \\
j \in\{1, \ldots, n+n v\} ; \\
k \in\{1, \ldots, m\} .
\end{array}\right.\right.
\end{aligned}
$$

A Função Objetivo do TDVRP-RT, Equação (1), representa a soma total dos horários de de regresso dos veículos ao depósito que deve ser minimizada. As Restrições (2), (3) e (4) asseguram que cada cliente seja visitado uma vez e exatamente $n v$ veículos sejam usados. As Restrições (5) definem o momento inicial de partida dos veículos do depósito nó 1 como maior ou igual a ti para todos os veículos. As Restrições (6) calculam o momento de partida de cada nó $j$ que corresponde aos clientes a serem atendidos. As Restrições (7) e (8) garantem que o arco representando o momento $k$ entre os nós $i$ e $j$ seja escolhido de acordo com o momento de partida do nó $i$.

As Restrições (9) impõem as janelas de tempo que são definidas em termos de momento de chegada aos nós enquanto as variáveis $t p_{i}$ representam o momento de partida dos nós. As Restrições (10), (11) e (12) impõem restrições de capacidade. As Restrições (11) asseguram que todos os veículos devem sair do depósito nó 1 com $\mathrm{q}_{\mathrm{i}}=0$, ou seja, sem entregar carga. As Restrições (10) asseguram a continuidade do descarregamento do veículo, o que significa que a carga descarregada pelo veículo que deixa o cliente $j$ é, pelo menos, igual à carga descarregada quando parte do cliente $i$ visitado anteriormente mais a carga entregue no cliente $j$.

As Restrições (12) garantem que a capacidade de cada veículo não seja ultrapassada. De forma análoga às Restrições (10), as Restrições (13) garantem a continuidade temporal das rotas, ou seja que momento de partida do veículo de um cliente, $t p_{i}$, seja maior ou igual ao momento mais cedo de saída do depósito, $t i$. As Restrições (14) garantem que a carga $q_{i}$ entregue pelo veículo que sai de um cliente seja maior ou igual a zero. As Restrições (15) definem que as variáveis $x_{i j}^{k}$ sejam binárias.

O TDVRP-RE também teve como base o TDVRP. Com o objetivo de se gerar rotas que promovam a redução da emissão de gases poluentes, o TDVRP-RT teve sua função objetivo alterada de forma que minimizasse as emissões de poluentes, e não o tempo total de regresso aos depósitos. Assim, o TDVRP-RE se diferencia do TDVRP-RT, pois, por meio dele, são obtidas rotas que visam menor poluição do meio ambiente.

Para o cálculo das emissões poluentes foi necessário buscar estudos que relacionassem a quantidade de gases liberados em função do tipo do veículo, da velocidade média percorrida e dos diversos fatores que caracterizam a via. As- 
sim, para este artigo foi utilizado o EMEP/EEA Air Pollutant Emission Inventory Guidebook (EMEP/EEA, 2013), que fornece orientações técnicas sobre emissões de poluentes. Segundo o guia, o processo de combustão dos motores dos veículos produz $\mathrm{CO}_{2}$ e $\mathrm{H}_{2} \mathrm{O}$ como principais produtos. A combustão também produz vários subprodutos que são originados pela oxidação incompleta do combustível (CO, hidrocarbonetos (HC), materiais particulados (PM)) ou da oxidação de espécies não combustíveis presentes na câmara de combustão (NOx).

Assim, novos parâmetros foram adicionadas ao modelo TDVRP-RE. São eles: $C O_{i j}^{k}, N O x_{i j}^{k}, H C_{i j}^{k}$ e $P M_{i j}^{k}$ que são, respectivamente, as quantidades em gramas emitidas de cada poluente no percurso do nó $i$ ao nó $j$ começando em $i$ durante o intervalo $k$, sendo $C O_{i i}^{k}=N O x_{i i}^{k}=H C_{i i}^{k}=$ $P M_{i i}^{k}=\infty$ para todo $i$.

Além dos parâmetros, novas variáveis de decisão foram adicionadas ao modelo TDVRP-RE: $C O p_{i}, N O x p_{i}$, $H C p_{i}$ e $P M p_{i}$, são, respectivamente, as quantidades acumuladas no momento de partida de algum veículo do nó $i$.

Assim, apresenta-se a seguir a função objetivo e as restrições do TDVRP-RE. Vale ressaltar, que o modelo TDVRP-RE é formado pela Função Objetivo, Equação (16), pelas Restrições (2) a (15), citadas anteriormente, e pelas Restrições (17) a (28).

Função objetivo:

$$
\operatorname{Min}\left(\sum_{v=1}^{n v} C O p_{n+v}+N O x p_{n+v}+H C p_{n+v}+P M p_{n+v}\right)
$$

sujeito às seguintes restrições:

$$
\begin{gathered}
C O p_{j}-C O p_{i}-B x_{i j}^{k} \geq C O_{i j}^{k}-B \\
\forall i, j, k \mid\left\{\begin{array}{l}
i \in\{1, \ldots, n\} ; \\
j \in\{2, \ldots, n+n v\} ; \\
i \neq j ; \\
m \in\{1, \ldots, m\}
\end{array}\right. \\
N O x p_{j}-N O p_{i}-B x_{i j}^{k} \geq N O x_{i j}^{k}-B
\end{gathered}
$$

$$
\begin{aligned}
& H C p_{1}=0 \\
& P M p_{1}=0 \\
& C O p_{i} \geq 0 \\
& N O x p_{i} \geq 0 \\
& H C p_{i} \geq 0 \\
& P M p_{i} \geq 0
\end{aligned}
$$

A Função Objetivo do TDVRP-RE, Equação (16), tem várias parcelas que representam a somatória de cada um dos gases emitidos e o que se busca é a redução de todas estas parcelas, ou seja, a redução de emissão total de poluentes das rotas. Esta função objetivo deve ser minimizada.

As Restrições (17) calculam a quantidade acumulada de $\mathrm{CO}$ emitida no nó $j$ que é igual à quantidade acumulada emitida no nó $i$ somada a quantidade em gramas emitida de CO no percurso do nó $i$ ao nó $j$ começando em $i$ durante o intervalo $k$. As Restrições (18) calculam a quantidade acumulada de NOx emitida no nó $j$ que é igual a quantidade acumulada emitida no nó $i$ somada a quantidade em gramas emitida de NOx no percurso do nó $i$ ao nó $j$ começando em $i$ durante o intervalo $k$. As Restrições (19) calculam a quantidade acumulada de HC emitida no nó $j$ que é igual à quantidade acumulada emitida no nó $i$ somada a quantidade em gramas emitida de HC no percurso do nó $i$ ao nó $j$ começando em $i$ durante o intervalo $k$. As Restrições (20) calculam a quantidade acumulada de PM emitida no nó $j$ que é igual à quantidade acumulada emitida no nó $i$ somada a quantidade em gramas emitida de PM no percurso do nó $i$ ao nó $j$ começando em $i$ durante o intervalo $k$.

As Restrições (21) a (24) garantem que as contagens das emissões sejam iniciadas em zero, a partir do depósito. $\mathrm{E}$ as Restrições (25) a (28) garantem que $C O p_{i}, N O x p_{i}$, $H C p_{i}$ e $P M p_{i}$ assumam valores maiores ou iguais a zero.

\section{APLICAÇÃO PRÁTICA}

Visando uma aplicação prática e em situações cotidianas, os modelos matemáticos TDVRP-RT e TDVRP-RE foram executados no pacote de otimização CPLEX 12.6 (IBM, 2016). Foram realizados testes com vários cenários para entendimento e avaliação dos resultados obtidos e, em seguida, para concepção de cenários reais, utilizaram-se dados de uma empresa que é uma rede varejista de hortifrutigranjeiros e que possui centros de distribuição que abastecem a região metropolitana de Vitória (ES). Dentre os clientes desta empresa estão algumas redes de supermercados, sendo uma dessas redes com 8 lojas designada para integrar os cenários reais. Assim, os dados inseridos no programa continham informações sobre:

- Veículos: A quantidade de veículos disponíveis para abastecimento dos oito supermercados da rede e suas capacidades dadas pela quantidade de paletes que podiam ser carregados pelos caminhões;

- Demanda dos supermercados: A carga foi caracterizada pela quantidade de paletes demandados pelos 
clientes, que deve ser sempre menor ou igual à capacidade dos caminhões.

- Tempos: Os tempos de viagem de um supermercado a outro ou de um supermercado ao depósito. O tempo de serviço em cada cliente, ou seja, o tempo de descarregamento, foi levantado junto à empresa e foi informado que era de quatro minutos para cada palete. Os intervalos de tempo em que os veículos podem chegar a cada um dos nós, definidos como o período de trabalho do motorista.

Além dessas considerações, apurou-se na região estudada por meio de pesquisa em campo que o dia pode ser dividido em três períodos diferenciados pelas condições de tráfego da região:

1. De $8 \mathrm{~h}$ às $10 \mathrm{~h}$, em que o veículo transita em velocidade média de $7 \mathrm{~km} / \mathrm{h}$ devido ao grande congestionamento;

2. De $10 \mathrm{~h}$ às $16 \mathrm{~h}$, em que o veículo transita em velocidade média de $35 \mathrm{~km} / \mathrm{h}$ devido às condições de trânsito livre;

3. De $16 \mathrm{~h}$ às $17 \mathrm{~h}$, em que o veículo transita em velocidade média de $20 \mathrm{~km} / \mathrm{h}$ devido congestionamento moderado.

Os fatores de emissão dependentes da velocidade de veículos pesados movidos a diesel são providos pelos padrões europeus para emissão de veículos. Devido ao grande número de dados requeridos para o cálculo das principais emissões (CO, HC, NOx, PM) e do consumo de combustível foram feitas algumas considerações, descritas a seguir.

Os veículos utilizados para atender as demandas dos supermercados são caminhões de serviços pesados, rígidos de 16 toneladas, tipo Euro V - SCR, movidos a diesel. Algumas simplificações do mundo real foram estabelecidas, tendo em vista a grande dificuldade de apura-las na prática. Em função da grande quantidade de ruas, não foram consideradas as inclinações das vias, ou seja, foi considerado que todas as ruas são planas. Um outro aspecto que não foi considerado é a relação do peso transportado com o consumo de combustível e para tal, considerou-se que a carga média seria de $50 \%$ da capacidade do veículo para levantamento dos parâmetros de emissão de poluentes, já que o veículo diminui sua carga à medida que abastece os supermercados por onde passa.. Assim, de acordo com estas considerações e com o guia definido por EMEP/EEA (2013), foram utilizadas as Equações (29) a (33) para o cálculo da quantidade de poluentes emitidos e do consumo de combustível (FC) em função da velocidade do veículo.

Nestas novas restrições, têm-se que $E_{C O}$ representa a quantidade emitida de $\mathrm{CO} ; E_{N O_{x}}$ representa a quantidade emitida de $\mathrm{NO}_{\mathrm{X}} ; \mathrm{E}_{\mathrm{HC}}$ representa a quantidade emitida de $\mathrm{HC} ; E_{P M}$ representa a quantidade emitida de $\mathrm{PM} ; E_{F C}$ representa a quantidade consumida de combustível e $V$ representa a velocidade do veículo.

$$
\begin{aligned}
& E_{C O}=0,764+18,374 /\left(1+e^{-1,514+1,362 \log (V)-0,003 V}\right) \\
& E_{N O_{x}}=66,533 \times 0,987^{V} \times V^{-0,580} \\
& E_{H C}=0,174-0,166 \times e^{-6,040 \times V^{-1,142}} \\
& E_{P M}=0,185-0,162 \times e^{-10,634 \times V^{-1,265}} \\
& E_{F C}=e^{7,430-0,340 / V-0,586 \log (V)}
\end{aligned}
$$

Além disso, como preconiza o EEMPE/EEA (2013), para ajustar o modelo aos valores de emissão para velocidades entre $12 \mathrm{~km} / \mathrm{h}$ e $86 \mathrm{~km} / \mathrm{h}$, foi necessário que a velocidade admitida no intervalo de $8 \mathrm{~h}$ às $10 \mathrm{~h}$ fosse de $12 \mathrm{~km} / \mathrm{h}$, em vez de $7 \mathrm{~km} / \mathrm{h}$. Após as considerações elencadas anteriormente, foi possível identificar a quantidade emitida de cada gás poluente em função das velocidades adotas neste estudo. Estes dados são apresentados na a Tabela 1.

Tabela 1. Quantidade emitida de gases poluentes em função da velocidade

\begin{tabular}{cccc} 
& \multicolumn{3}{c}{ Velocidade Percorrida (Intervalo Dia) } \\
\cline { 2 - 4 } Gases & $\mathbf{1 2 k m} / \mathbf{h}$ & $\mathbf{3 5 k m} / \mathbf{h}$ & $\mathbf{2 0 k m} / \mathbf{h}$ \\
Poluentes & $\mathbf{( 8 h}$ às 10h) & $(\mathbf{1 0 h}$ às 16h) & $\mathbf{( 1 6 h ~ a ̀ s ~ 1 7 h ) ~}$ \\
\hline $\mathrm{CO}$ & $10,322 \mathrm{~g} / \mathrm{km}$ & $7,774 \mathrm{~g} / \mathrm{km}$ & $9,048 \mathrm{~g} / \mathrm{km}$ \\
$\mathrm{NOx}$ & $13,489 \mathrm{~g} / \mathrm{km}$ & $5,393 \mathrm{~g} / \mathrm{km}$ & $9,049 \mathrm{~g} / \mathrm{km}$ \\
$\mathrm{HC}$ & $0,057 \mathrm{~g} / \mathrm{km}$ & $0,057 \mathrm{~g} / \mathrm{km}$ & $0,037 \mathrm{~g} / \mathrm{km}$ \\
$\mathrm{PM}$ & $0,083 \mathrm{~g} / \mathrm{km}$ & $0,083 \mathrm{~g} / \mathrm{km}$ & $0,057 \mathrm{~g} / \mathrm{km}$ \\
\hline
\end{tabular}

Com estes dados foi possível obter os valores de emissão no percurso de um nó a outro em função do intervalo do dia. A seguir são apresentados os valores para cada parâmetro. Foram considerados nove nós, sendo oito clientes a serem atendidos mais a origem e nove veículos disponíveis para o atendimento dos clientes.

Três intervalos de tempo foram considerados para cada ligação entre os nós, $8 \mathrm{~h}$ às $10 \mathrm{~h}, 10 \mathrm{~h}$ às $16 \mathrm{~h}$ e $16 \mathrm{~h}$ às $17 \mathrm{~h}$, ou seja, quatro momentos no dia. Assim, o momento 0 é o momento inicial, considerado às 8 horas. Os momentos 1,2 e 3 marcam o término dos intervalos do dia considerados: o momento 1 é às 10 horas, o momento 2 é às 16 horas e o momento 3 é às 17 horas. $\mathrm{O}$ momento de partida do depósito de origem é às 8 horas da manhã, o momento mais cedo para chegar em cada nó é às 8 horas da manhã, e o momento mais tarde para chegar em cada nó é às 17 horas da tarde.

A capacidade de carregamento dos veículos é de 16 paletes. A quantidade de carga (em paletes) entregue a cada cliente é diferente em cada cenário e é apresentada na Tabela 2. O tempo para entregar cada palete é de quatro minutos, portanto, o tempo de serviço (em horas) em cada nó é calculado como sendo o número de paletes demandados em cada nó vezes quatro minutos, Tabela 3 . O tempo de viagem de um nó a outro, em cada intervalodo dia, (em horas) é calculado por meio da divisão da distância (em quilômetros) de um nó a outro pela velocidade praticada no intervalo do dia que o veículo está. A distância em quilometros se encontra na Tabela 4. A velocidade considerada para cada momento foi, respectivamente, $12 \mathrm{~km} / \mathrm{h}, 35 \mathrm{~km} / \mathrm{h}$ e $20 \mathrm{~km} / \mathrm{h}$.

Para cada tipo de poluente (CO, NOx, HC e PM) foram calculadas as emissões em cada trecho por meio da multiplicação das distâncias $(\mathrm{km})$ de um nó a outro pelos parâmetros de emissão de cada poluente $(\mathrm{g} / \mathrm{km})$ para cada velocidade praticada (Tabela 1 ).

Tabela 2. Demandas de cada cliente para cada cenário

\begin{tabular}{lccccc} 
& Cenário & Cenário & Cenário & Cenário & Cenário \\
& $\mathbf{1}$ & $\mathbf{2}$ & $\mathbf{3}$ & $\mathbf{4}$ & $\mathbf{5}$ \\
\hline Cliente 1 & 13 & 6 & 8 & 4 & 6 \\
Cliente 2 & 3 & 9 & 6 & 6 & 7 \\
Cliente 3 & 10 & 12 & 14 & 10 & 10 \\
Cliente 4 & 6 & 6 & 6 & 6 & 6 \\
Cliente 5 & 6 & 12 & 11 & 8 & 8 \\
Cliente 6 & 6 & 7 & 7 & 4 & 6 \\
Cliente 7 & 6 & 7 & 8 & 7 & 6 \\
Cliente 8 & 6 & 9 & 11 & 7 & 8 \\
\hline
\end{tabular}


Tabela 3. Tempo de serviço em cada cliente para cada cenário em horas

\begin{tabular}{lccccc} 
& Cenário 1 & Cenário 2 & Cenário 3 & Cenário 4 & Cenário 5 \\
\hline Cliente 1 & 0,87 & 0,40 & 0,53 & 0,27 & 0,40 \\
Cliente 2 & 0,20 & 0,60 & 0,40 & 0,40 & 0,47 \\
Cliente 3 & 0,67 & 0,80 & 0,93 & 0,67 & 0,67 \\
Cliente 4 & 0,40 & 0,40 & 0,40 & 0,40 & 0,40 \\
Cliente 5 & 0,40 & 0,80 & 0,73 & 0,53 & 0,53 \\
Cliente 6 & 0,40 & 0,47 & 0,47 & 0,27 & 0,40 \\
Cliente 7 & 0,40 & 0,47 & 0,53 & 0,47 & 0,40 \\
Cliente 8 & 0,40 & 0,60 & 0,73 & 0,47 & 0,53 \\
\hline
\end{tabular}

Tabela 4. Distâncias de um nó a outro

\begin{tabular}{|c|c|c|c|c|c|c|c|c|c|}
\hline & Depósito & Cliente 1 & Cliente 2 & Cliente 3 & Cliente 4 & Cliente 5 & Cliente 6 & Cliente 7 & Cliente 8 \\
\hline Depósito & 0 & 5.2 & 5.7 & 5.7 & 12.4 & 13.5 & 13.3 & 17.1 & 8.2 \\
\hline Cliente 1 & 4.5 & 0 & 4.9 & 14.9 & 11.0 & 11.0 & 11.0 & 14.6 & 5.5 \\
\hline Cliente 2 & 5.2 & 4.4 & 0 & 10.4 & 6.5 & 8.7 & 8.5 & 12.3 & 3.2 \\
\hline Cliente 3 & 5.2 & 16.7 & 12.5 & 0 & 6.0 & 9.1 & 9.5 & 10.0 & 13.8 \\
\hline Cliente 4 & 13.5 & 12.3 & 8.1 & 3.9 & 0 & 10.0 & 10.4 & 10.9 & 9.5 \\
\hline Cliente 5 & 13.4 & 11.3 & 9.6 & 7.1 & 8.8 & 0 & 1.1 & 4.0 & 5.9 \\
\hline Cliente 6 & 13.6 & 11.5 & 9.8 & 7.5 & 9.2 & 1.5 & 0 & 5.1 & 6.1 \\
\hline Cliente 7 & 17.0 & 15.0 & 13.3 & 10.9 & 12.6 & 5.1 & 5.1 & 0 & 9.5 \\
\hline Cliente 8 & 8.2 & 6.1 & 4.4 & 12.6 & 9.0 & 5.6 & 5.4 & 9.2 & 0 \\
\hline
\end{tabular}

$n v_{\text {total }}$ - representa o número total de veículos que fo-

\section{RESULTADOS}

Foram gerados por meio do solver CPLEX 12.6 resultados para 5 cenários, tanto no TDVRP-RT, como no TDVRP-RE. Depois de gerados os resultados, estes foram organizados e apresentados na Tabela 5 e na Tabela 6 para fins de comparação. Na Tabela 5, são apresentados os resultados referentes à utilização dos veículos, i.e, quantidade de veículos utilizada, capacidade total, capacidade utilizada e capacidade disponível dos veículos. Estes resultados foram os mesmos para os dois modelos, TDVRP-RT e TDVRP-RE.

Tabela 5. Resultados da utilização dos veículos

\begin{tabular}{lcccc} 
& $\begin{array}{c}\text { Veículos } \\
\text { Utilizados }\end{array}$ & $\begin{array}{c}\text { Capacidade } \\
\text { Total }\end{array}$ & $\begin{array}{c}\text { Capacidade } \\
\text { Utilizada }\end{array}$ & $\begin{array}{c}\text { Capacidade } \\
\text { Disponível }\end{array}$ \\
\hline Cenário 1 & 4 & 64 & 56 & 8 \\
Cenário 2 & 5 & 80 & 68 & 12 \\
Cenário 3 & 6 & 96 & 71 & 25 \\
Cenário 4 & 4 & 64 & 52 & 12 \\
Cenário 5 & 4 & 64 & 57 & 7 \\
\hline
\end{tabular}

Nota-se que, em todos os cenários, os veículos não foram utilizados completamente, o que representaria para a empresa uma falha no aproveitamento de recursos. Isto ocorre devido a impossibilidade de descarregamento fracionado para os clientes, já que só podem ser visitados uma vez e por somente um veículo, toda a caga demanda deve ser entregue de uma vez. Os roteamentos apresentados mostram que os trajetos realizados pelos veículos não sofreram grandes variações de um modelo para o outro. No entanto, no TDVRP-RE todos os trajetos são realizados do segundo intervalo do dia em que a velocidade do percurso é maior. A pouca variação nas rotas pode ser justificada pela baixa combinação de possibilidades para ocupação dos veículos conforme explicado anteriormente.

$\mathrm{Na}$ Tabela 6 foram analisados os indicadores listados a seguir: ram utilizados para atender as demandas dos clientes e indica também a quantidade de rotas (uma para cada veículo);

$t v_{\text {total }}$ - representa a soma dos tempos de viagem de todas as rotas;

$d_{\text {total }}$ - representa a distância total percorrida por todos os veículos utilizados;

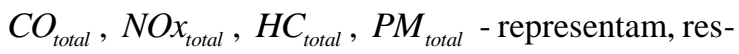
pectivamente, as quantidades totais emitidas por todos os veículos utilizados de $\mathrm{CO}, \mathrm{NOx}, \mathrm{HC}$ e PM;

$F C_{\text {total }}$ - representa o total de combustível consumido por todos os veículos utilizados; $T R_{\text {total }}$ - representa a soma dos momentos de regresso ao depósito dos veículos utilizados; $T R_{\text {méd }}$, - representa a média dos momentos de regresso ao depósito;

$T R_{\text {máx }}$ e $T R_{\text {mín }}$ - representam, respectivamente, o maior e o menor momento de regresso ao depósitos das rotas geradas.

Os dados da Tabela 5 mostram que, para todos os cenários, a quantidade de veículos utilizados é a mesma nos dois modelos, isto porque o que determina o número de veículos são suas capacidades e as demandas de cada nó, que não se alteram. Isto significa que não haveria gastos com eventuais aumentos da frota de veículos da empresa para os cenários estudados. Em relação às distâncias totais, verifica-se que no modelo com foco no meio ambiente ocorreu um aumento no Cenário 3 e pequenas reduções nos demais cenários. Estas pequenas variações ocorreram, pois as distâncias não são consideradas nas funções objetivos dos modelos.

No TDVRP-RE, é possível notar também que ocorreram reduções consideráveis no tempo total de viagem, em todas as quantidades de poluentes emitidos e nos consumos de combustível. Estas reduções podem ser consideradas como vantagens do TDVRP-RE por também diminuir os custos nesses itens. As reduções das emissões já eram esperadas, justamente, por serem o objetivo do TDVRP-RE. 
Tabela 6. Resultados das variáveis analisadas

\begin{tabular}{|c|c|c|c|c|c|c|}
\hline & \multirow{2}{*}{\multicolumn{3}{|c|}{ Cenário 1}} & \multirow{2}{*}{\multicolumn{3}{|c|}{ Cenário 2}} \\
\hline & & & & & & \\
\hline & TDVRP-RT & TDVRP-RE & Diferença (\%) & TDVRP-RT & TDVRP-RE & Diferença (\%) \\
\hline$n v_{\text {total }}$ & 4 & 4 & 0.00 & 5 & 5 & 0.00 \\
\hline$t v_{\text {total }}(\mathrm{h})$ & 15.64 & 11.85 & -24.27 & 11.50 & 7.90 & -31.32 \\
\hline$d_{\text {total }}(\mathrm{km})$ & 179.83 & 169.63 & -5.67 & 117.80 & 117.70 & -0.08 \\
\hline$C O_{\text {total }}(\mathrm{g})$ & 1561.07 & 1317.98 & -15.57 & 1082.85 & 914.53 & -15.54 \\
\hline$N O x_{\text {total }}(\mathrm{g})$ & 1477.11 & 914.28 & -38.56 & 1167.11 & 634.40 & -45.64 \\
\hline$H C_{\text {total }}(\mathrm{g})$ & 6.97 & 3.39 & -51.36 & 4.98 & 2.36 & -52.61 \\
\hline$P M_{\text {total }}(\mathrm{g})$ & 10.02 & 6.78 & -32.34 & 7.34 & 4.72 & -35.69 \\
\hline$F C_{\text {total }}(\mathrm{g})$ & 134058.83 & 114664.80 & -14.47 & 92456.93 & 79564.02 & -13.94 \\
\hline$T R_{\text {total }}(\mathrm{h})$ & 47.67 & 51.85 & 8.06 & 51.70 & 57.90 & 10.71 \\
\hline$T R_{\text {méd }}(\mathrm{h})$ & 11.92 & 13.03 & 8.57 & 10.34 & 11.60 & 10.85 \\
\hline$T R_{\text {máx }}(\mathrm{h})$ & 13.67 & 14.69 & 6.93 & 10.97 & 10.97 & 8.16 \\
\hline \multirow[t]{3}{*}{$T R_{\min }(\mathrm{h})$} & 10.81 & 11.97 & 9.67 & 9.71 & 9.71 & 12.61 \\
\hline & \multicolumn{3}{|c|}{ Cenário 3} & \multicolumn{3}{|c|}{ Cenário 4} \\
\hline & TDVRP-RT & TDVRP-RE & Diferença $(\%)$ & TDVRP-RT & TDVRP-RE & Diferença $(\%)$ \\
\hline$n v_{\text {total }}$ & 6 & 6 & 0.00 & 4 & 4 & 0.00 \\
\hline$t v_{\text {total }}(\mathrm{h})$ & 12.69 & 8.30 & -34.54 & 9.35 & 6.22 & -33.40 \\
\hline$d_{\text {total }}(\mathrm{km})$ & 125.40 & 127.00 & 1.26 & 101.10 & 96.50 & -4.55 \\
\hline $\operatorname{CO}_{\text {total }}(\mathrm{g})$ & 1268.66 & 971.25 & -23.44 & 924.78 & 755.86 & -18.27 \\
\hline$N O x_{\text {total }}(\mathrm{g})$ & 1316.14 & 673.95 & -48.79 & 987.19 & 536.61 & -45.64 \\
\hline$H C_{\text {total }}(\mathrm{g})$ & 6.22 & 2.50 & -59.81 & 4.20 & 2.02 & -51.90 \\
\hline$P M_{\text {total }}(\mathrm{g})$ & 8.93 & 5.02 & -43.78 & 6.23 & 3.95 & -36.60 \\
\hline$F C_{\text {total }}(\mathrm{g})$ & 108013.47 & 84498.85 & -21.77 & 79001.06 & 65673.01 & -16.87 \\
\hline$T R_{\text {total }}(\mathrm{h})$ & 61.05 & 68.30 & 10.61 & 41.38 & 46.22 & 10.47 \\
\hline$T R_{\text {méd }}(\mathrm{h})$ & 10.18 & 11.38 & 10.62 & 10.35 & 11.58 & 10.67 \\
\hline$T R_{m a ́ x}(\mathrm{~h})$ & 11.01 & 12.01 & 8.34 & 10.88 & 11.99 & 9.23 \\
\hline \multirow[t]{3}{*}{$T R_{\min }(\mathrm{h})$} & 9.34 & 10.81 & 13.60 & 9.31 & 10.71 & 13.08 \\
\hline & \multicolumn{3}{|c|}{ Cenário 5} & \multirow{2}{*}{\multicolumn{3}{|c|}{ Legenda }} \\
\hline & TDVRP-RT & TDVRP-RE & Diferença $(\%)$ & & & \\
\hline$n v_{\text {total }}$ & 4 & 4 & 0.00 & \multicolumn{3}{|c|}{$n v_{\text {total }} \rightarrow$ número de veículos usados } \\
\hline tv $v_{\text {total }}(\mathrm{h})$ & 10.25 & 6.69 & -34.75 & \multicolumn{3}{|c|}{ tv $v_{\text {total }}(\mathrm{h}) \rightarrow$ tempo total de viagem } \\
\hline$d_{\text {total }}(\mathrm{km})$ & 101.50 & 97.80 & -3.65 & \multicolumn{3}{|c|}{$d_{\text {total }}(\mathrm{km}) \rightarrow$ distância total percorrida } \\
\hline$C O_{\text {total }}(\mathrm{g})$ & 935.02 & 759.91 & -18.73 & \multicolumn{3}{|c|}{$\mathrm{CO}_{\text {total }}(\mathrm{g}) \rightarrow$ quantidade emitida de $\mathrm{CO}$} \\
\hline NOx total $(\mathrm{g})$ & 1012.03 & 527.15 & -47.91 & \multicolumn{3}{|c|}{$N O x_{\text {total }}(\mathrm{g}) \rightarrow$ quantidade emitida de NOx } \\
\hline$H C_{\text {total }}(\mathrm{g})$ & 4.33 & 1.95 & -54.97 & \multicolumn{3}{|c|}{$H C_{\text {total }}(\mathrm{g}) \rightarrow$ quantidade emitida de $\mathrm{HC}$} \\
\hline$P M_{\text {total }}(\mathrm{g})$ & 6.36 & 3.91 & -38.52 & \multicolumn{3}{|c|}{$P M_{\text {total }}(\mathrm{g}) \rightarrow$ quantidade emitida de PM } \\
\hline$F C_{\text {total }}(\mathrm{g})$ & 79818.04 & 66111.83 & -17.17 & \multicolumn{3}{|c|}{$F C_{\text {total }}(\mathrm{g}) \rightarrow$ consumo total de combustível } \\
\hline$T R_{\text {total }}(\mathrm{h})$ & 41.87 & 46.69 & 10.32 & \multicolumn{3}{|c|}{$T R_{\text {total }}(\mathrm{h}) \rightarrow$ momento de retorno total } \\
\hline$T R_{\text {méd }}(\mathrm{h})$ & 10.47 & 11.81 & 11.40 & \multicolumn{3}{|c|}{$T R_{\text {méd }}(\mathrm{h}) \rightarrow$ momento de retorno médio } \\
\hline$T R_{\text {máx }}(\mathrm{h})$ & 10.88 & 12.28 & 11.37 & \multicolumn{3}{|c|}{$T R_{\text {máx }}(\mathrm{h}) \rightarrow$ maior momento de retorno } \\
\hline$T R_{\min }(\mathrm{h})$ & 10.10 & 11.38 & 11.25 & \multicolumn{3}{|c|}{$T R_{\min }(\mathrm{h}) \rightarrow$ menor momento de retorno } \\
\hline
\end{tabular}

Como as emissões têm relação diretamente proporcional com o consumo de combustível, suas reduções irão, consequentemente, diminuir as quantidades de combustível consumido. Outra redução interessante foi a redução do tempo total de viagem, que ocorre porque todas as rotas são iniciadas e realizadas no intervalo de tempo em que as condições são de trânsito livre e os veículos percorrem os trajetos com velocidades maiores.

O TDVRP-RT não conseguiu melhores tempos totais de viagem por causa da sua função objetivo, que visa reduzir o momento de retorno ao depósito e não o tempo total de viagem. Fazer com que as rotas tivessem menor tempo de viagem, ou seja, fazer com que fossem realizadas no segundo intervalo, em que velocidades são maiores, provocaria o aumento no momento de regresso ao depósito, o que transgride a sua função objetivo. Portanto, o TDVRP-RT busca, primeiramente, realizar todas as entregas e regressar ao depósito o quanto antes e, depois, busca a forma mais rápida, enquanto que o TDVRP-RE não faz considerações em relação ao momento de retorno ao depósito.

Desta forma, a desvantagem do modelo que visa diminuir a quantidade de poluentes emitidos é que para conseguir as reduções apresentadas o início das entregas foi feito mais tarde, o que, na prática, poderia ser representado por tempos de espera e tempos operacionais ociosos ou desperdiçados. Para demandas maiores, ter que começar a fazer as entregas mais tarde também poderia implicar no aumento da frota para que todas as entregas fossem feitas no intervalo de tempo que permitisse velocidades maiores. Além disso, podem existir casos no qual o cliente pode não estar disponível para receber entregas no intervalo que proporciona redução das emissões de poluentes.

Por fim, este artigo apresentou dois modelos matemáticos TDVRP-RT e TDVRP-RE e analisou os resultados de ambos e apesar de não ser o senso comum atualmente, buscar o objetivo ambiental pode trazer ganhos financeiros para a empresa, no entanto, estes resultados precisam ser analisados sob a ótica do nível de serviço do cliente, ou seja, se ele pode estar disponível no horário que seja mais ambientalmente limpo. Além disso, para o TDVRP-RE alcançar seus bons resultados, ele teve que gerar atrasos na saída dos caminhões do depósito e, assim, gerou horários de ociosidade do veículo, o que pode levar a uma incapacidade futura de atender a todos os clientes, ocasionando assim a necessidade de investimento em mais frota e eventualmente mais emissão de poluentes. 


\section{CONCLUSÕES}

Para avaliar o impacto de adotar uma estratégia ambiental no planejamento da logística nas empresas que operam com logística urbana, foi feita a comparação dos resultados da aplicação de dois modelos matemáticos propostos neste artigo baseados no TDVRP nos quais foram utilizados dados de uma empresa de distribuição de alimentos. Por serem baseados no TDVRP, os dois modelos consideram que a velocidade com que os veículos circulam numa região metropolitana depende do momento do dia em que se realiza o transporte de mercadorias devido às condições de tráfego.

O modelo denominado TDVRP-RT tem como objetivo a redução do tempo para realização das entregas de mercadoria e sua função objetivo é a redução da soma dos momentos de regresso ao depósito dos veículos utilizados. O outro modelo, denominado TDVRP-RE, foi uma adaptação do TDVRP-RT que considera questões ambientais e, então, seu objetivo visa a redução da emissão de poluentes e sua função objetivo é reduzir a soma das quantidades de poluentes emitidos (CO, NOx, HC e PM).

A fim de comparar as duas estratégias, foram utilizados dados de uma empresa distribuidora de alimentos de uma região metropolitana. Os itens dos resultados comparados foram a quantidade de veículos, suas capacidades e rotas utilizados para o transporte, o tempo total de viagem dos veículos, a distância total percorrida, as quantidades totais emitida de $\mathrm{CO}$, NOx, HC e PM, o consumo total de combustível, o momento total de retorno ao depósito, o momento médio de retorno ao depósito, o maior e menor momento de retorno ao depósito.

Dos demais itens verificados, o tempo total de viagem, as quantidades totais emitidas e o consumo total de combustível do TDVRP-RE tiveram valores menores quando comparados aos do TDVRP-RT, nas execuções dos 5 cenários testados. Na prática, estas reduções proporcionariam diminuições nos custos relacionados a estes pontos para empresas que realizam esse tipo de transporte. No entanto, os momentos de regresso ao depósito nos resultados do TDVRP-RE aumentaram significativamente em relação aos do TDVRP-RT. Isso denota que para que essas economias fossem possíveis, o início do transporte deveria ser atrasado para que os veículos percorressem as rotas com velocidades maiores, emitindo menos poluentes, o que poderia significar desperdício e ociosidade de tempo e baixo aproveitamento dos motoristas. Além disso, eventualmente os clientes podem não estar disponíveis para recebimento de carga nos intervalos para o transporte com menor emissão de poluentes. Desta forma, cabe à empresa a escolha do modelo que se adéqua mais à sua estratégia.

Este artigo apresentou dois modelos matemáticos TDVRP-RT e TDVRP-RE e analisou os resultados de ambos e apesar de não ser o senso comum atualmente, buscar o objetivo ambiental pode trazer ganhos financeiros para a empresa, no entanto, estes resultados precisam ser analisados sob a ótica do nível de serviço do cliente, ou seja, se ele pode estar disponível no horário que seja mais ambientalmente limpo. Além disso, para o TDVRP-RE alcançar seus bons resultados, ele teve que gerar atrasos na saída dos caminhões do depósito e, assim, gerou horários de ociosidade do veículo, o que pode levar a uma incapacidade futura de atender a todos os clientes, ocasionando assim a necessidade de investimento em mais frota e eventualmente mais emissão de poluentes.

Os modelos obtiveram resultados para cenários de pequeno porte. Assim, é também importante analisar os resultados em um cenário com maior número de clientes a serem atendidos a fim de verificar situações onde os veículos fossem completamente carregados. Além disso, é necessário considerar mais fatores, como a inclinação das vias, a variação da frota de veículos, a variação do peso do veículo devido à coleta/entrega das mercadorias durante os percursos. A inclusão de frota heterogênea também seria um estudo importante para a logística urbana, visto que cada tipo de veículo gera diferentes quantidades de emissões de poluentes. Assim, para casos em que, por exemplo, seja necessária uma viagem em horários de tráfego intenso, um veículo menor poderia ser empregado com a finalidade de poluir menos. Ou então, a definição do melhor tipo de veículo poderia também ser influenciada pelas condições da via, como sua inclinação.

Como sugestão de trabalhos futuros, sugere-se que todos os itens mencionados anteriormente e não tratados neste artigo, sejam incorporados ao modelo e novos testes sejam realizados. Além disso, seria interessante propor meta-heurísticas para os dois modelos propostos visando resolver instâncias de maior porte.

\section{AGRADECIMENTOS}

Os autores agradecem à FAPES (Proc. 458/2013e75528452/2016) e ao CNPq (Proc. 477357/2013-0) pelo apoio financeiro.

\section{REFERÊNCIAS}

BEKTAŞ, Tolga; LAPORTE, Gilbert. The pollution-routing problem. Transportation Research Part B: Methodological, v. 45, n. 8, p. 1232-1250, 2011. DOI: 10.1016/j.trb.2011.02.004

DONATI, Alberto V. et al. Time dependent vehicle routing problem with a multi ant colony system. European journal of operational research, v. 185 , n. 3, p. 1174-1191, 2008. DOI: 10.1016/j.ejor.2006.06.047

ERDOĞAN, Sevgi; MILLER-HOOKS, Elise. A green vehicle routing problem. Transportation Research Part E: Logistics and Transportation Review, v. 48, n. 1, p. 100-114, 2012. DOI: 10.1016/j.tre.2011.08.001

EEA, EMEP. EEA air pollutant emission inventory guidebook, Technical guidance to prepare national emission inventories, Part B - 2013. European Environment Agency (EEA), Copenhagen, 2013.

ERICSSON, Eva; LARSSON, Hanna; BRUNDELL-FREIJ, Karin. Optimizing route choice for lowest fuel consumptionpotential effects of a new driver support tool. Transportation Research Part C: Emerging Technologies, v. 14, n. 6, p. 369-383, 2006. DOI: $10.1016 /$ j.trc.2006.10.001

FLEISCHMANN, Bernhard; GIETZ, Martin; GNUTZMANN, Stefan. Time-varying travel times in vehicle routing. Transportation science, v. 38, n. 2, p. 160-173, 2004. DOI: $10.1287 /$ trsc. 1030.0062 
HILL, Arthur V.; BENTON, W. C. Modelling intra-city timedependent travel speeds for vehicle scheduling problems. Journal of the Operational Research Society, v. 43, n. 4, p. 343-351, 1992. DOI: $10.1057 /$ jors. 1992.49

IBM. (2016) IBM ILOG CPLEX Optimization Studio. Disponível em: http://www-

03.ibm.com/software/products/en/ibmilogcpleoptistud. Acesso em: 10 de Março de 2016.

ICHOUA, Soumia; GENDREAU, Michel; POTVIN, Jean-Yves. Vehicle dispatching with time-dependent travel times. European journal of operational research, v. 144, n. 2, p. 379-396, 2003. DOI: $10.1016 / \mathrm{S} 0377-2217(02) 00147-9$

JABALI, O.; WOENSEL, T.; DE KOK, A. G. Analysis of travel times and $\mathrm{CO} 2$ emissions in time- dependent vehicle routing. Production and Operations Management, v. 21, n. 6, p. 10601074, 2012. DOI: 10.1111/j.1937-5956.2012.01338.x

KIRBY, Howard R. et al. Modelling the effects of transport policy levers on fuel efficiency and national fuel consumption. Transportation Research Part D: Transport and Environment, v. 5, n. 4, p. 265-282, 2000. DOI:10.1016/S1361-9209(99)00037-1

KUCUKOGLU, I. et al. Green capacitated vehicle routing problem fuel consumption optimization model. Computational Engineering Research, v. 3, p. 16-23, 2013.

LAPORTE, Gilbert. What you should know about the vehicle routing problem. Naval Research Logistics (NRL), v. 54, n. 8, p. 811-819, 2007. DOI: $10.1002 /$ nav.20261

LIN, Canhong et al. Survey of green vehicle routing problem: past and future trends. Expert Systems with Applications, v. 41, n. 4, p. 1118-1138, 2014. DOI: 10.1016/j.eswa.2013.07.107

MALANDRAKI, Chryssi; DASKIN, Mark S. Time dependent vehicle routing problems: Formulations, properties and heuristic algorithms. Transportation science, v. 26, n. 3, p. 185-200, 1992. DOI: $10.1287 /$ trsc.26.3.185

MALANDRAKI, Chryssi; DIAL, Robert B. A restricted dynamic programming heuristic algorithm for the time dependent traveling salesman problem. European Journal of Operational Research, v. 90, n. 1, p. 45-55, 1996. DOI: 10.1016/03772217(94)00299-1

NANNICINI, Giacomo et al. Fast paths in large-scale dynamic road networks. Computational Optimization and Applications, v. 45, n. 1, p. 143-158, 2010. DOI:10.1007/s10589-008-9172-y

PALMER, Andrew. The development of an integrated routing and carbon dioxide emissions model for goods vehicles. $\mathrm{PhD}$ thesis, Cranfield University, School of Management, 2007.

SCOTT, Cathy; URQUHART, Neil; HART, Emma. Influence of topology and payload on $\mathrm{CO} 2$ optimised vehicle routing. In: European Conference on the Applications of Evolutionary Computation. Springer Berlin Heidelberg, 2010. p. 141-150. DOI: 10.1007/978-3-642-12242-2_15

TANIGUCHI, Eiichi; THOMPSON, Russell G. City Logistics Network Modelling and Intelligent Transport Systems. 2001.

VAN WOENSEL, Tom; VANDAELE, Nico. Empirical validation of a queueing approach to uninterrupted traffic flows. 4OR, v. 4, n. 1, p. 59-72, 2006.

VAN WOENSEL, Tom.; CRETEN, Ruth; VANDAELE, Nico. Managing the environmental externalities of traffic logistics: The issue of emissions. Production and Operations Management, v. 10 , n. 2, p. 207-223, 2001. DOI: $10.1111 /$ j.19375956.2001.tb00079.x

VAN WOENSEL, Tom et al. Vehicle routing with dynamic travel times: A queueing approach. European journal of operational research, v. 186, n. 3, p. 990-1007, 2008. DOI: 10.1016/j.ejor.2007.03.012 